\title{
Surgical outcomes following encephaloduroarteriosynangiosis in adult moyamoya disease associated with Type 2 diabetes
}

\author{
*Bin Ren, BS, ${ }^{1,2}$ Zheng-Shan Zhang, MM, ${ }^{2}$ Wei-Wei Liu, MM, ${ }^{3}$ Xiang-Yang Bao, MM, ${ }^{2}$ \\ De-Sheng Li, MD, ${ }^{2}$ Cong Han, MM, ${ }^{2}$ Peng Xian, MM, ${ }^{2}$ Feng Zhao, BS, ${ }^{2}$ Hui Wang, BS, ${ }^{2}$ \\ Hai Wang, MD, PhD, ${ }^{1}$ and Lian Duan, MD, PhD²
}

${ }^{1}$ Cardiovascular Drug Research Center, Institute of Health and Environmental Medicine, Academy of Military Medical Sciences; and ${ }^{2}$ Department of Neurosurgery, 307th Hospital of PLA, Center for Cerebral Vascular Disease; and ${ }^{3}$ Consulting Centre of Biomedical Statistics, Academy of Military Medical Sciences, Beijing, China

\begin{abstract}
OBJECTIVE Debate exists regarding the merits and shortcomings of an indirect bypass procedure for treating adult patients with moyamoya disease (MMD). Considerable variation in neovascularization occurs among different organs in patients with diabetes mellitus. Here, the effect of encephaloduroarteriosynangiosis on MMD associated with Type 2 diabetes mellitus (T2DM) is evaluated.
\end{abstract}

METHODS A retrospective and 1:2 matched case-control study was conducted in moyamoya patients with or without T2DM $(n=180)$. Postoperative collateral formations were graded according to the Modified Collateral Grading System that originated from the Matsushima Angiographic Stage Classification. Neurological function outcomes before and after the operation were evaluated according to the modified Rankin Scale. Univariate and multivariate logistic regression analyses were performed to determine the risk factors for clinical outcome.

RESULTS There was no statistically significant difference in the constituent ratios of initial symptom and preoperative Suzuki stage between patients with and without T2DM. Progression of angiopathy around the circle of Willis was postoperatively observed in bilateral internal carotid arteries in both groups. Patients with T2DM had a higher postoperative Suzuki stage $(p<0.01)$ and more frequent development of collateral angiogenesis germinating from the external carotid after indirect revascularization procedures in the surgical cerebral hemisphere $(82.7 \%$ vs $72.2 \% ; p<0.05)$. The extent of postoperative collateral formation in patients with diabetes mellitus was significantly higher $(p<0.01)$. Postoperative clinical improvement in the diabetes group was more common after revascularization procedures $(p<0.05)$, and the diabetes group had lower modified Rankin Scale scores $(p<0.05)$ in comparison with the nondiabetes group. Late postoperative stroke and posterior cerebral artery involvement were identified as predictors of unfavorable clinical outcome in both groups, while T2DM was associated with a favorable clinical outcome.

CONCLUSIONS Encephaloduroarteriosynangiosis is an efficacious treatment for adult patients with MMD. Patients with T2DM could achieve better collateral circulation and clinical improvement following surgery.

http://thejns.org/doi/abs/10.3171/2015.7.JNS15218

KEY WORDS moyamoya disease; Type 2 diabetes; revascularization; collaterals; clinical outcome; vascular disorders

$\mathrm{M}$ OYAMOYA disease (MMD), a cerebral vascular disease, is characterized by progressive stenosis and occlusion of the terminal portion of the bilateral internal carotid arteries, leading to the formation of an abnormal vascular network composed of collateral pathways at the base of the brain. MMD can cause a reduc- tion in the blood supply to the brain, and there are 2 major etiological categories of symptoms: those due to brain ischemia (i.e., stroke, transient ischemic attack, and seizure) and those due to the deleterious consequences of the compensatory mechanisms in response to ischemia (i.e., hemorrhage from fragile collateral vessels and headache

ABBREVIATIONS CBF = cerebral blood flow; $\mathrm{DM}=$ diabetes mellitus; $\mathrm{EDAS}=$ encephaloduroarteriosynangiosis; $\mathrm{MCA}=$ middle cerebral artery; $\mathrm{MMD}=$ moyamoya disease; $\mathrm{mRS}=$ modified Rankin Scale; PCA = posterior cerebral artery; $\mathrm{STA}=$ superficial temporal artery; T2DM = Type $2 \mathrm{DM}$.

SUBMITTED January 29, 2015. ACCEPTED July 28, 2015.

INCLUDE WHEN CITING Published online January 8, 2016; DOI: 10.3171/2015.7.JNS15218.

* Drs. Hai Wang and Lian Duan contributed equally to this work. 
from dilated transdural collaterals) ${ }^{27}$ Surgical revascularizations such as direct, indirect, or combined anastomosis have been proven to be effective in improving decreased cerebral circulation and clinical signs. ${ }^{25}$ Encephaloduroarteriosynangiosis (EDAS) is an indirect revascularization procedure that has been shown to be beneficial for pediatric $^{6,12,22}$ and adult populations. ${ }^{2,5,7}$ The results of revascularization due to EDAS are associated with some factors, including age, complications, and other unknown factors.

Patients with diabetes mellitus (DM) tend to develop more severe events in cerebrovascular and cardiovascular diseases. ${ }^{4,8,13}$ Different outcomes of neovascularization attributed to DM have been found in various organ systems. ${ }^{1,10,19,26}$ The effect of DM on cerebral neovascularization remains unclear. In this study, we sought to investigate the effect of EDAS on clinical outcomes and explore the distinction of collateral circulation between MMD patients with and without type 2 diabetes mellitus (T2DM).

\section{Methods \\ Patient Selection}

A consecutive surgical series of patients with MMD who underwent EDAS between April 2004 and December 2013 at the Department of Neurosurgery, 307th Hospital of PLA, Beijing, China, was reviewed to identify all patients 18 years and older at the time of surgery. All patients were diagnosed as having MMD based on cerebral digital subtraction angiography findings. Inclusion and exclusion criteria were set in accordance with the criteria for the diagnosis and treatment of MMD. ${ }^{9}$ We divided all MMD patients into 2 groups: those with T2DM and those without T2DM. All T2DM patients had received a definite diagnosis of T2DM according to the World Health Organization's definition. ${ }^{3}$ Sixty-one MMD patients with T2DM fulfilled the inclusion criteria above, but a female patient was excluded because she underwent neural stem cell transplantation after the operation. Finally, 60 MMD patients with T2DM composed the study group. Fifty-three patients with T2DM received medical intervention (36 patients took oral hypoglycemic agents, 7 patients were administered insulin, and 10 patients underwent combination therapy), and 7 patients adopted diet control before admission and during follow-up. By using a matched-pair case-control study design, 120 control participants were randomly selected and matched according to age and sex from the MMD patients without T2DM.

\section{Retrospective Chart Review}

Clinical records, including hospital charts, clinic notes, and radiological studies, were reviewed. ${ }^{7}$ All data were collected through March 2015. The study protocol was approved by the institutional ethics committee.

\section{Angiographic Evaluation}

Cerebral angiography was routinely performed within 1 to 2 weeks before surgery. The angiographic stages of MMD were estimated according to the Suzuki Angiographic Stage Classification. ${ }^{33}$ We routinely checked the digital subtraction angiograms postoperatively in all patients (with or without T2DM) to assess collateral circula- tion in the superficial temporal artery (STA) to the operated hemispheres. Based on the classification proposed by Matsushima et al., ${ }^{22}$ our angiographic outcomes were further graded into 4 degrees according to the extent of the blood supply: excellent, in which the area supplied by the surgical bypass covered over two-thirds of the middle cerebral artery (MCA) distribution; good, in which between twothirds and one-third of the MCA distribution was covered; fair, in which less than one-third of the cortical branch of the MCA was covered through the indirect bypass; and poor, in which no collateral circulation was observed.

To eliminate bias, 2 independent and blinded interventional radiologists were enrolled in this research to analyze the results of cerebral angiography.

\section{Surgical Technique}

All patients underwent an EDAS procedure. Revascularization was performed based on the patient's symptoms, cerebral blood flow (CBF) studies, angiographic findings, and dominant side if no lateralizing signs or symptoms appeared. The original EDAS procedure with a posterior branch of the STA was performed. The course of the STA was mapped by an experienced neurosurgeon in order to identify the bifurcation preoperatively. The skin was incised over the STA, and a fascial strip measuring 7 to 8 $\mathrm{cm} \times 1 \mathrm{~cm}$ and containing the artery was meticulously dissected. Bur holes were placed, and a small bone window craniotomy was performed along the superior temporal line. The dura was opened, and the fascial strip measuring 6 to $7 \mathrm{~cm} \times 1 \mathrm{~cm}$ was sutured to the edges of the linear dural incision. The bone flap was replaced with ample space at the bur holes for the vessel. ${ }^{21} \mathrm{~A}$ contralateral procedure was routinely performed 3 months after the first surgery.

\section{Clinical Follow-Up}

Long-term outcome was ascertained via clinical visits and telephone. Our clinical outcomes were divided into 4 grades: 1) excellent, in which the preoperative symptoms had totally disappeared with no fixed neurological deficits; 2) good, in which the symptoms had markedly decreased but neurological deficits remained; 3) fair, in which the symptoms persisted though less frequently; and 4) poor, in which the preoperative status remained either unchanged or worsened or new symptoms appeared..$^{20,28}$ The modified Rankin Scale (mRS) was used to determine the neurological functional outcomes before and after operation.

Perioperative stroke was defined as either infarction or hemorrhage present on MR or CT imaging that developed during the surgery or within 1 month after the surgery. Late postoperative stroke was defined as such an event occurring during follow-up. Postoperative conditions and long-term outcomes were ascertained through the last clinical visit if the patients were unable to be contacted.

\section{Statistical Analysis}

Unordered categorical variables were evaluated using the chi-square test or Fisher exact test, including the baseline data, operative effects, and late postoperative stroke during follow-up. The Wilcoxon signed-rank test was used to assess the changes in Suzuki stages and mRS between 
TABLE 1. Clinical comparison of the diabetes and nondiabetes groups*

\begin{tabular}{|c|c|c|c|}
\hline Characteristic & $\begin{array}{l}\text { Diabetes } \\
\text { Group } \\
(n=60)\end{array}$ & $\begin{array}{c}\text { Nondiabetes } \\
\text { Group } \\
(n=120)\end{array}$ & p Value \\
\hline Mean age $\pm S D$ & $42.08 \pm 8.20$ & $41.33 \pm 8.42$ & 0.568 \\
\hline Female sex & $21(35.0)$ & $42(35.0)$ & 1.000 \\
\hline Smoking & $13(21.7)$ & $20(16.7)$ & 0.414 \\
\hline Drinking & $13(21.7)$ & $15(12.5)$ & 0.110 \\
\hline Hypertension & $31(51.7)$ & $45(37.5)$ & 0.070 \\
\hline Hyperlipidemia & $17(28.3)$ & $24(21.7)$ & 0.209 \\
\hline Initial symptom & & & 0.471 \\
\hline TIA & $23(38.3)$ & $42(35.0)$ & \\
\hline Cerebral infarction & $19(31.7)$ & $29(24.2)$ & \\
\hline $\begin{array}{l}\text { Intracerebral hemor- } \\
\text { rhage }\end{array}$ & $5(8.3)$ & $22(18.3)$ & \\
\hline Headache & $8(13.3)$ & $15(12.5)$ & \\
\hline Dizziness & $3(5.0)$ & $9(7.5)$ & \\
\hline Involuntary movements & $1(1.7)$ & $1(0.8)$ & \\
\hline Asymptomatic & $1(1.7)$ & $2(1.7)$ & \\
\hline PCA involvement & $32(53.3)$ & $50(41.7)$ & 0.138 \\
\hline Admission mRS score & & & 0.694 \\
\hline 0 & $1(1.7)$ & $2(1.7)$ & \\
\hline 1 & $35(58.3)$ & $73(60.8)$ & \\
\hline 2 & $19(31.7)$ & $38(31.7)$ & \\
\hline 3 & $4(6.7)$ & $5(4.2)$ & \\
\hline 4 & $1(1.7)$ & $2(1.7)$ & \\
\hline 5 & $0(0.0)$ & $0(0.0)$ & \\
\hline 6 & $0(0.0)$ & $0(0.0)$ & \\
\hline
\end{tabular}

$\mathrm{TIA}=$ transient ischemic attack.

* Values are given as the number of patients (\%) unless otherwise noted.

preoperation and postoperation in the same group. Meanwhile, the extent of the collateral formation, changes in $\mathrm{mRS}$ scores, and improvement of clinical symptoms were analyzed using the Wilcoxon rank-sum test. Univariate and multivariate logistic regression analyses were performed to determine the risk factors for clinical outcomes. Differences were considered statistically significant at $\mathrm{p}<$ 0.05 . All statistical analyses were carried out using SPSS software for Windows (version 20.0; IBM).

\section{Results \\ Patient Population}

One hundred eighty patients with MMD were analyzed in our cohort, and the clinical baseline characteristics are summarized in Table 1 . In the diabetes group, only 21 $(35.0 \%)$ patients were female. The most common initial symptom in both groups was cerebral ischemia, such as transient ischemic attack and cerebral infarction. Thirtysix patients $(60.0 \%)$ demonstrated a slight advantage with an initial $\mathrm{mRS}<2$, in contrast to 75 patients $(62.5 \%)$ in the nondiabetes group, though this was not statistically different. Thirty-two patients with T2DM were observed with steno-occlusive changes of the posterior cerebral artery
TABLE 2. Preoperative and postoperative Suzuki stage between the 2 groups*

\begin{tabular}{cccccc}
\hline \multirow{2}{*}{$\begin{array}{c}\text { Suzuki } \\
\text { Stage }\end{array}$} & \multicolumn{2}{c}{ Preoperative Suzuki Stage } & & \multicolumn{2}{c}{ Postoperative Suzuki Stage } \\
\cline { 2 - 3 } \cline { 5 - 5 } & Diabetes & Nondiabetes & & Diabetest & Nondiabetes \\
\hline 1 & $8(6.7)$ & $30(12.5)$ & & $6(5.0)$ & $18(7.5)$ \\
\hline 2 & $12(10.0)$ & $18(7.5)$ & & $3(2.5)$ & $21(8.8)$ \\
\hline 3 & $22(18.4)$ & $39(16.2)$ & & $12(10.0)$ & $24(10.0)$ \\
\hline 4 & $46(38.3)$ & $84(35.0)$ & & $26(21.7)$ & $76(31.6)$ \\
\hline 5 & $16(13.3)$ & $48(20)$ & $37(30.8)$ & $70(29.2)$ \\
\hline 6 & $16(13.3)$ & $21(8.8)$ & $36(30.0)$ & $31(12.9)$ \\
\hline Total & 120 & 240 & 120 & 240 \\
\hline
\end{tabular}

* Values are given as the number of hemispheres (\%); both hemispheres were evaluated for all patients.

$\dagger p<0.01$ versus the nondiabetes group.

(PCA) in comparison with 50 patients in the nondiabetes group $(\mathrm{p}>0.05)$.

\section{Angiographic Outcomes}

There were no statistical differences observed in the timing of postoperative angiography. The mean follow-up period lasted for a duration of $10.17 \pm 4.63$ months (range $6-24$ months) in the diabetes group and $10.41 \pm 4.93$ months (range 6-25 months) in the nondiabetes group. No statistically significant differences in preoperative Suzuki stage were observed between the 2 groups ( $p>$ 0.05 ). Meanwhile, we found further progression of angiopathy around the circle of Willis postoperatively in both groups, respectively. Nevertheless, patients with T2DM had a much higher postoperative Suzuki stage than patients without T2DM $(\mathrm{p}<0.01)$ (Table 2).

The development and extension of STA-leptomeninges collateral circulation after surgery were illustrated in Table 3. Patients with T2DM were more likely to develop neovascularization in the surgical cerebral hemisphere $(82.7$ vs $72.2 \% ; p<0.05)$. In addition, statistically significant differences were observed between the 2 groups with regard to the extent grade of the collateral circulation $(\mathrm{p}<0.01)$ (Table 3).

\section{Surgical Treatment}

All patients underwent EDAS procedures for surgical treatment. Fifty bilateral and 10 right-sided procedures were performed in the T2DM group. Meanwhile, 120

TABLE 3. Collateral formation on the operative hemisphere between the 2 groups*

\begin{tabular}{lcc}
\hline Collateral Formation & Diabetest & Nondiabetes \\
\hline Poor (none) & $19(17.3)$ & $59(27.8)$ \\
\hline Fair $(<1 / 3$ of MCA) & $20(18.2)$ & $52(24.5)$ \\
\hline Good $(2 / 3$ to $1 / 3$ of MCA) & $50(45.4)$ & $71(33.5)$ \\
\hline Excellent $(>2 / 3$ of MCA) & $21(19.1)$ & $30(14.2)$ \\
\hline Total & 110 & 212 \\
\hline
\end{tabular}

\footnotetext{
* Values are given as the number of operative hemispheres (\%).
}

$\dagger p<0.01$ versus the nondiabetes group. 
TABLE 4. Distribution of clinical outcomes between 2 groups*

\begin{tabular}{ccc}
\hline Clinical Outcome & Diabetes Group $\dagger$ & Nondiabetes Group \\
\hline Poor & $5(8.3)$ & $16(13.3)$ \\
\hline Fair & $3(5.0)$ & $7(5.8)$ \\
\hline Good & $17(28.3)$ & $48(40.0)$ \\
\hline Excellent & $35(58.3)$ & $49(40.8)$ \\
\hline Total & 60 & 120 \\
\hline
\end{tabular}

\footnotetext{
* Values are given as the number of patients (\%).

$\dagger p<0.05$ versus the nondiabetes group.
}

nondiabetic patients underwent 92 bilateral, 11 right-sided, and 17 left-sided procedures in the nondiabetes group. Acute cerebral infarction during the perioperative period occurred in 1 patient in each group.

\section{Results of Follow-Up}

The median duration of follow-up was 45.65 months (range 8-131 months). The mean follow-up period lasted for a duration of $44.92 \pm 26.13$ months (range 9-131 months) in the diabetic cohort and $46.02 \pm 26.13$ months (range 8-111 months) in the nondiabetes group, which was not statistically different $(p>0.05)$. The clinical outcomes in MMD patients with T2DM demonstrated statistical difference $(\mathrm{p}<0.05)$ (Table 4). While no statistical difference had been observed for clinical outcomes among different treatments of T2DM in the diabetic group ( $\mathrm{p}>0.05)$. Overall 13 patients suffered late postoperative strokes during close follow-up. Three patients with ischemic stroke (at 8 months, 48 months, and 54 months postoperatively) and 1 patient with hemorrhagic stroke (3 months postoperatively) were observed in the diabetes group. Five patients had 1 ischemic stroke and 4 patients had 1 intracerebral hemorrhage in the nondiabetes group. No statistically significant difference was detected for stroke occurrence or recurrent hemorrhage during the follow-up between the comparable series $(p>0.05)$. No fatal case was shown in MMD patients with T2DM; nevertheless, 2 of the nondiabetic patients (who initially presented with hemorrhage) died of brain rebleeding.

Univariate logistic regression analyses of the preoperative clinical variables showed that MMD was associated with T2DM (OR 1.919; 95\% CI 1.054-3.496; $p=0.034$ ) and identified as a predictor of favorable clinical outcome, while late postoperative stroke (OR 0.022; 95\% CI $0.006-0.085 ; \mathrm{p}=0.000$ ) and PCA involvement (OR 0.543; $95 \%$ CI 0.311-0.948; $p=0.032$ ) were associated with unfavorable clinical outcomes (Table 5). Multivariate logistic regression analyses of the preoperative clinical variables revealed that patients with T2DM (OR 2.132; 95\% CI $1.123-4.047 ; \mathrm{p}=0.021$ ) were associated with a favorable clinical outcome. Late postoperative stroke (OR 0.020; 95\% CI $0.005-0.078 ; \mathrm{p}=0.000$ ) and PCA involvement (OR 0.530; 95\% CI 0.293-0.959; $\mathrm{p}=0.036$ ) were identified as predictors of adverse clinical outcomes (Table 6).

Most of the patients in both groups experienced dramatic mRS improvement. More favorable outcome on mRS was observed in the diabetic MMD patients with a statistically significant difference between the groups ( $\mathrm{p}<$ 0.05) (Table 7).
TABLE 5. Univariate logistic regression analyses for predictive factors of clinical outcome

\begin{tabular}{|c|c|c|c|}
\hline Variable & OR & $95 \% \mathrm{Cl}$ & $\mathrm{p}$ Value \\
\hline Age at onset & 1.019 & $0.986-1.054$ & 0.251 \\
\hline \multicolumn{4}{|l|}{ Sex } \\
\hline Female & 1 & & \\
\hline Male & 1.362 & $0.761-2.438$ & 0.297 \\
\hline \multicolumn{4}{|l|}{ Smoking } \\
\hline No & 1 & & \\
\hline Yes & 0.669 & $0.332-1.349$ & 0.262 \\
\hline \multicolumn{4}{|l|}{ Drinking } \\
\hline No & 1 & & \\
\hline Yes & 0.737 & $0.348-1.562$ & 0.425 \\
\hline \multicolumn{4}{|l|}{ Hypertension } \\
\hline No & 1 & & \\
\hline Yes & 1.031 & $0.591-1.800$ & 0.912 \\
\hline \multicolumn{4}{|c|}{ Associated w/ T2DM } \\
\hline No & 1 & & \\
\hline Yes & 1.919 & $1.054-3.496$ & 0.034 \\
\hline \multicolumn{4}{|l|}{ Hyperlipidemia } \\
\hline No & 1 & & \\
\hline Yes & 1.031 & $0.541-1.966$ & 0.925 \\
\hline \multicolumn{4}{|l|}{ TIA } \\
\hline No & 1 & & \\
\hline Yes & 1.350 & $0.759-2.402$ & 0.308 \\
\hline \multicolumn{4}{|l|}{ Infarction } \\
\hline No & 1 & & \\
\hline Yes & 0.925 & $0.498-1.718$ & 0.804 \\
\hline \multicolumn{4}{|l|}{ Hemorrhage } \\
\hline No & 1 & & \\
\hline Yes & 0.615 & $0.288-1.313$ & 0.210 \\
\hline \multicolumn{4}{|l|}{ Headache } \\
\hline No & 1 & & \\
\hline Yes & 0.564 & $0.251-1.268$ & 0.167 \\
\hline \multicolumn{4}{|l|}{ Dizziness } \\
\hline No & 1 & & \\
\hline Yes & 1.296 & $0.422-3.975$ & 0.650 \\
\hline \multicolumn{4}{|l|}{ Asymptomatic } \\
\hline No & 1 & & \\
\hline Yes & 2.606 & $0.228-29.793$ & 0.441 \\
\hline \multicolumn{4}{|c|}{ PCA involvement } \\
\hline No & 1 & & \\
\hline Yes & 0.543 & $0.311-0.948$ & 0.032 \\
\hline \multicolumn{4}{|c|}{ Late postop stroke } \\
\hline No & 1 & & \\
\hline Yes & 0.022 & $0.006-0.085$ & 0.000 \\
\hline
\end{tabular}

\section{Discussion}

MMD is a chronic and progressive cerebral vascular disease with increasing prevalence. At present, 2 common approaches, indirect and direct revascularization, are recommended for the augmentation of CBF in MMD 
TABLE 6. Multivariate logistic regression analyses for predictive factors of clinical outcome

\begin{tabular}{lccc}
\hline \multicolumn{1}{c}{ Variable } & OR & $95 \% \mathrm{Cl}$ & $\mathrm{p}$ Value \\
\hline T2DM & 2.132 & $1.123-4.047$ & 0.021 \\
\hline PCA involvement & 0.530 & $0.293-0.959$ & 0.036 \\
\hline Late postop stroke & 0.020 & $0.005-0.078$ & 0.000 \\
\hline
\end{tabular}

patients. Meanwhile, combined revascularization is performed in some neurosurgery departments. ${ }^{25}$ There is wide controversy regarding the merits and shortcomings of the 2 surgical approaches for adults with MMD., ${ }^{2,5,7,23,31}$ Starke et al. reviewed the literature concerning the surgical treatment and concluded that both direct and indirect bypass were effective. ${ }^{30}$ Since there is a lack of randomized trials, it was noted that the selection of the surgical modalities depended on the preference and surgical technique mastered by the cerebrovascular neurosurgeon. ${ }^{17}$ Furthermore, it is well known that patients with DM tend to develop stroke events in cerebrovascular and cardiovascular diseases. ${ }^{4,8,13}$ In our cohort, all adult patients underwent EDAS. Adequate pial collateral vessel formation, and improvement in clinical outcomes and mRS scores, in addition to a reduced number of stroke events, were observed in both groups in our study. Thus, we conclude that EDAS is an effective procedure for adult MMD patients with and without T2DM. Furthermore, our multivariate logistic regression analyses also showed that T2DM was a favorable prognostic factor for clinical outcome following EDAS in MMD.

Previous studies attempted to identify the prognostic factors for postoperative complications and clinical outcomes. ${ }^{5,6,11,14,32,34}$ Preoperative cerebral infarction, younger age at onset, involvement of the PCA, intentional hypotension, high Suzuki stage, and postoperative ischemic events have been observed to correlate with postoperative complications and outcomes. According to the multivariate logistic regression analyses, our study showed that PCA involvement in both groups with no statistically significant differences preoperatively together with the late postoperative stroke was associated with unfavorable clinical outcomes, which was similar to the previous results observed in our center. ${ }^{5}$ We speculate that PCA stenoses may influence regional CBF and cerebrovascular reactivity and then impair cerebral perfusion, which could lead to ischemic symptoms postoperatively. However, the limited number of patients and lack of evaluation on blood flow and metabolism before and after the surgery restrict our further research. Therefore, a unified assessment method for perfusion is needed.

Different outcomes of neovascularization attributed to DM have been found in various organ systems. Excessive angiogenesis exists in diabetic retinopathy and impaired angiogenesis in coronary and peripheral circulations. ${ }^{1,10,19,26}$ In our study, there was more frequent collateral formation in the diabetes group. Greater extent of collaterals (more than one-third of the MCA distribution) was also detected in patients with T2DM. We deduce that T2DM could promote pial collateral vessel formation in patients with chronic cerebral ischemia, such as MMD.
TABLE 7. $m R S$ assessment during follow-up*

\begin{tabular}{ccc}
\hline$m$ RS Score & Diabetes Group & Nondiabetes Group \\
\hline 0 & $33(55.0)$ & $48(45.0)$ \\
\hline 1 & $21(35.0)$ & $44(36.7)$ \\
\hline 2 & $1(1.7)$ & $22(18.3)$ \\
\hline 3 & $5(8.3)$ & $1(0.8)$ \\
\hline 4 & $0(0.0)$ & $2(1.7)$ \\
\hline 5 & $0(0.0)$ & $0(0.0)$ \\
\hline 6 & $0(0.0)$ & $3(2.5)$ \\
\hline Total & 60 & \\
\hline$*$ Values are given as the number of patients $(\%)$. & \\
$\dagger p<0.05$ versus the nondiabetes group.
\end{tabular}

The mechanism of neovascularization affected by DM in various organs remains unclear. Li et al. had confirmed that angiogenesis and arteriogenesis showed remarkable increases in the number of collaterals, the diameters of the collaterals, the number of anastomoses, and microvessel density in T2DM Goto-Kakizaki rats. ${ }^{16}$ The experimental results revealed a significant rise in the level of vascular endothelial growth factor (VEGF) and nitrotyrosine. ${ }^{24}$ The levels of growth factors and cytokines that were regarded as important in the development of sprouting collateral vessels from the extracranial vascular system by indirect revascularization, such as the expression of VEGF, basic fibroblast growth factor, transforming growth factor$\beta$, granulocyte colony-stimulating factor, and hepatocyte growth factor, have been proven to be elevated in many studies in MMD patients. ${ }^{35}$ Therefore, we propose the following hypothesis: the expression of growth factors and cytokines described above might be higher in MMD patients complicated with T2DM than in MMD patients without T2DM. Thus, more formation regarding collateral angiogenesis can be detected in MMD patients with T2DM.

Changes in the Suzuki stage, which were demonstrated to continuously progress postoperatively, were observed in both groups in our cohort. This means that the progression of angiopathy around the circle of Willis existed in our study just as other literature had reported..$^{15,18,29,36}$ However, patients with T2DM demonstrated much higher postoperative Suzuki stages than nondiabetic patients. That is, more rapid progression of angiopathy around the circle of Willis existed in patients with T2DM. We speculate that aggravated cerebral ischemia caused by the progression of angiopathy might promote more neovascularization in MMD patients complicated with T2DM after indirect revascularization.

Several limitations have been found in the present study. First, the number of the T2DM patients in this retrospective study was small, and the patients in the nondiabetes group were matched only for age and sex. All potential confounding factors could not be controlled, and some influential factors were not taken into account. Therefore, studies on the relationship between collateral circulation and other risk factors are needed in order to provide further estimates. Second, analyses of CBF and metabolism were absent before and after the operations in our study. 
We did not adopt this approach due to the lack of quantitative data after neuroradiological examination in our center. Meanwhile, imaging methods for evaluating cerebral hemodynamics were different in our cases. Some patients underwent CBF SPECT, CT perfusion, and MR perfusion assessments, while others underwent PET evaluation. Therefore, a prospective study with a unified assessment method for perfusion is needed. Third, the lack of animal models of MMD may be the greatest obstacle to exploring the mechanisms for etiology, pathology, and pathophysiology involved in the collateral circulation between T2DM and nondiabetic MMD patients. Thus, we cannot perform a basic study to interpret our findings now.

\section{Conclusions}

The present study reveals that EDAS is an efficacious treatment for adult patients with MMD. Patients with MMD with T2DM had more favorable prognosis and more collateral circulations following EDAS. Further prospective study and basic research need to be performed to investigate the factors affecting formation of the collateral vessels in MMD patients associated with T2DM.

\section{Acknowledgments}

This study was supported by a grant from National Natural Science Foundation of China (no. 81171083). The funders had no role in the study design, data collection and analysis, decision to publish, or preparation of the manuscript.

\section{References}

1. Abaci A, Oğuzhan A, Kahraman S, Eryol NK, Unal S, Arinç $\mathrm{H}$, et al: Effect of diabetes mellitus on formation of coronary collateral vessels. Circulation 99:2239-2242, 1999

2. Agarwalla PK, Stapleton CJ, Phillips MT, Walcott BP, Venteicher AS, Ogilvy CS: Surgical outcomes following encephaloduroarteriosynangiosis in North American adults with moyamoya. J Neurosurg 121:1394-1400, 2014

3. Alberti KG, Zimmet PZ: Definition, diagnosis and classification of diabetes mellitus and its complications. Part 1: diagnosis and classification of diabetes mellitus provisional report of a WHO consultation. Diabet Med 15:539-553, 1998

4. Almdal T, Scharling H, Jensen JS, Vestergaard H: The independent effect of type 2 diabetes mellitus on ischemic heart disease, stroke, and death: a population-based study of 13,000 men and women with 20 years of follow-up. Arch Intern Med 164:1422-1426, 2004

5. Bao XY, Duan L, Yang WZ, Li DS, Sun WJ, Zhang ZS, et al: Clinical features, surgical treatment and long-term outcome in adult patients with moyamoya disease in China. Cerebrovasc Dis 34:305-313, 2012

6. Bao XY, Duan L, Yang WZ, Li DS, Sun WJ, Zhang ZS, et al: Clinical features, surgical treatment, and long-term outcome in pediatric patients with moyamoya disease in China. Cerebrovasc Dis 39:75-81, 2015

7. Duan L, Bao XY, Yang WZ, Shi WC, Li DS, Zhang ZS, et al: Moyamoya disease in China: its clinical features and outcomes. Stroke 43:56-60, 2012

8. Ergul A, Kelly-Cobbs A, Abdalla M, Fagan SC: Cerebrovascular complications of diabetes: focus on stroke. Endocr Metab Immune Disord Drug Targets 12:148-158, 2012

9. Fukui M: Guidelines for the diagnosis and treatment of spontaneous occlusion of the circle of Willis ('moyamoya' disease). Research Committee on Spontaneous Occlusion of the Circle of Willis (Moyamoya Disease) of the Ministry of Health and Welfare, Japan. Clin Neurol Neurosurg 99 (Suppl 2):S238-S240, 1997

10. Hammes HP, Feng Y, Pfister F, Brownlee M: Diabetic retinopathy: targeting vasoregression. Diabetes 60:9-16, 2011

11. Huang AP, Liu HM, Lai DM, Yang CC, Tsai YH, Wang KC, et al: Clinical significance of posterior circulation changes after revascularization in patients with moyamoya disease. Cerebrovasc Dis 28:247-257, 2009

12. Ishikawa T, Houkin K, Kamiyama H, Abe H: Effects of surgical revascularization on outcome of patients with pediatric moyamoya disease. Stroke 28:1170-1173, 1997

13. Jeerakathil T, Johnson JA, Simpson SH, Majumdar SR: Short-term risk for stroke is doubled in persons with newly treated type 2 diabetes compared with persons without diabetes: a population-based cohort study. Stroke 38:1739-1743, 2007

14. Kim SH, Choi JU, Yang KH, Kim TG, Kim DS: Risk factors for postoperative ischemic complications in patients with moyamoya disease. J Neurosurg 103 (5 Suppl):433-438, 2005

15. Kim YO, Joo SP, Seo BR, Rho YI, Yoon W, Woo YJ: Early clinical characteristics according to developmental stage in children with definite moyamoya disease. Brain Dev 35:569-574, 2013

16. Li W, Prakash R, Kelly-Cobbs AI, Ogbi S, Kozak A, ElRemessy AB, et al: Adaptive cerebral neovascularization in a model of type 2 diabetes: relevance to focal cerebral ischemia. Diabetes 59:228-235, 2010

17. Lin N, Aronson JP, Manjila S, Smith ER, Scott RM: Treatment of Moyamoya disease in the adult population with pial synangiosis. J Neurosurg 120:612-617, 2014

18. Lin N, Baird L, Koss M, Kopecky KE, Gone E, Ullrich NJ, et al: Discovery of asymptomatic moyamoya arteriopathy in pediatric syndromic populations: radiographic and clinical progression. Neurosurg Focus 31 (6):E6, 2011

19. Lizotte F, Paré M, Denhez B, Leitges M, Guay A, Geraldes P: PKCס impaired vessel formation and angiogenic factor expression in diabetic ischemic limbs. Diabetes 62:2948-2957, 2013

20. Matsushima T, Fujiwara S, Nagata S, Fujii K, Fukui M, Kitamura K, et al: Surgical treatment for paediatric patients with moyamoya disease by indirect revascularization procedures (EDAS, EMS, EMAS). Acta Neurochir (Wien) 98:135-140, 1989

21. Matsushima Y, Fukai N, Tanaka K, Tsuruoka S, Inaba Y, Aoyagi M, et al: A new surgical treatment of moyamoya disease in children: a preliminary report. Surg Neurol 15:313320, 1981

22. Matsushima T, Inoue T, Suzuki SO, Fujii K, Fukui M, Hasuo $\mathrm{K}$ : Surgical treatment of moyamoya disease in pediatric patients-comparison between the results of indirect and direct revascularization procedures. Neurosurgery 31:401-405, 1992

23. Mizoi K, Kayama T, Yoshimoto T, Nagamine Y: Indirect revascularization for moyamoya disease: is there a beneficial effect for adult patients? Surg Neurol 45:541-549, 1996

24. Prakash R, Somanath PR, El-Remessy AB, Kelly-Cobbs A, Stern JE, Dore-Duffy P, et al: Enhanced cerebral but not peripheral angiogenesis in the Goto-Kakizaki model of type 2 diabetes involves VEGF and peroxynitrite signaling. Diabetes 61:1533-1542, 2012

25. Research Committee on the Pathology and Treatment of Spontaneous Occlusion of the Circle of Willis: Guidelines for diagnosis and treatment of moyamoya disease (spontaneous occlusion of the circle of Willis). Neurol Med Chir (Tokyo) 52:245-266, 2012

26. Ruiter MS, van Golde JM, Schaper NC, Stehouwer CD, Huijberts MS: Diabetes impairs arteriogenesis in the periph- 
eral circulation: review of molecular mechanisms. Clin Sci (Lond) 119:225-238, 2010

27. Scott RM, Smith ER: Moyamoya disease and moyamoya syndrome. N Engl J Med 360:1226-1237, 2009

28. Seol HJ, Wang KC, Kim SK, Hwang YS, Kim KJ, Cho BK: Headache in pediatric moyamoya disease: review of 204 consecutive cases. J Neurosurg 103 (5 Suppl):439-442, 2005

29. Smith ER, Scott RM: Progression of disease in unilateral moyamoya syndrome. Neurosurg Focus 24 (2):E17, 2008

30. Starke RM, Komotar RJ, Connolly ES: Optimal surgical treatment for moyamoya disease in adults: direct versus indirect bypass. Neurosurg Focus 26 (4):E8, 2009

31. Starke RM, Komotar RJ, Hickman ZL, Paz YE, Pugliese AG, Otten ML, et al: Clinical features, surgical treatment, and long-term outcome in adult patients with moyamoya disease. Clinical article. J Neurosurg 111:936-942, 2009

32. Strother MK, Anderson MD, Singer RJ, Du L, Moore RD, Shyr Y, et al: Cerebrovascular collaterals correlate with disease severity in adult North American patients with moyamoya disease. AJNR Am J Neuroradiol 35:1318-1324, 2014

33. Suzuki J, Kodama N: Moyamoya disease-a review. Stroke 14:104-109, 1983

34. Togao O, Mihara F, Yoshiura T, Tanaka A, Noguchi T, Kuwabara Y, et al: Cerebral hemodynamics in moyamoya disease: correlation between perfusion-weighted MR imaging and cerebral angiography. AJNR Am J Neuroradiol 27:391-397, 2006

35. Weinberg DG, Arnaout OM, Rahme RJ, Aoun SG, Batjer
$\mathrm{HH}$, Bendok BR: Moyamoya disease: a review of histopathology, biochemistry, and genetics. Neurosurg Focus 30 (6):E20, 2011

36. Yeon JY, Shin HJ, Kong DS, Seol HJ, Kim JS, Hong SC, et al: The prediction of contralateral progression in children and adolescents with unilateral moyamoya disease. Stroke 42:2973-2976, 2011

\section{Disclosures}

The authors report no conflict of interest concerning the materials or methods used in this study or the findings specified in this paper.

\section{Author Contributions}

Conception and design: Duan, Ren, Hai Wang. Acquisition of data: Ren. Analysis and interpretation of data: Ren, Liu. Drafting the article: Ren, Zhang. Reviewed submitted version of manuscript: Duan, Zhang, Hai Wang. Approved the final version of the manuscript on behalf of all authors: Duan. Statistical analysis: Liu. Administrative/technical/material support: Xian, Zhao, Hui Wang. Study supervision: Bao, Li, Han.

\section{Correspondence}

Lian Duan, Department of Neurosurgery, 307th Hospital of PLA, Center for Cerebral Vascular Disease, 8 Dong-Da St., Fengtai District, Beijing 100071, China. email: duanlian307@sina.com. 